\title{
Dynamic Vision Sensor Based High Efficiency Device Control
}

\author{
B. Praveena, H. Shravani, A. Shivani
}

\begin{abstract}
To control a specific device hardware switches and gesture based controls are usually used. There are some inconveniences involved in these controlling mechanism aging problem, safety related issues, high replacement cost in hardware switches and the impact of external noise in case of gesture based control. And in order to overcome these issues a new device control mechanism is brought up using two dimensional hologram and dynamic vision sensor. This involves reduced hardware usage and if needed, the controlling unit can be replaced easily. It possesses high processing efficiency and speed. The light source used in hologram processing unit is LED and it contributes less power consumption. The conventional proximity sensors are replaced by Dynamic vision sensor because the conventional sensors process series of frames continuously which is inefficient because it contains redundant information, wasting energy computational power and time. This drawback is eliminated by using DVS which starts to process the frames only when any change is sensed only at the time the change occurs. Proximity sensors are more prone to external noise whereas DVS is not. It has high signal to noise ratio.
\end{abstract}

Keywords: DVS; FDAPA; UART; CSMA; PSNR; Human machine interface; $S$-MAC

\section{INTRODUCTION}

In existing home appliances hardware switches or gesture devices are used to control the specific application. We need to allocate specific places to fix that hardware switch thus creating safety related issues, so we need to protect the children from the hardware. If we want to replace a device, we need to change the total wiring section; also we cannot change the position or place of the switch in an easy way. Replacement cost of the wiring or switch is more. Aging problem may occur due to continuous usage of the hardware switches. In the Gesture based control method lighting conditions, Real time interaction and Gesture vocabulary related issues may be there, thus making inconvenience to user to control the devices. In order to overcome these problems, a new device control mechanism is brought up using 2-D hologram [1] and in order to reduce power consumption LED is used as light source. Conventional proximity sensors are replaced by dynamic vision sensor because it has the fastest response time.

Revised Manuscript Received on September 27, 2019.

* Correspondence Author

B. Praveena, Assistant Professor, Department of Computer Science and Engineering, RMK Engineering College, Chennai. Email: praveena15secure@gmail.com

H. Shravani, Department of Computer Science and Engineering, RMK Engineering College, Chennai.

A. Shivani, Department of Computer Science and Engineering, RMK Engineering College, Chennai.
Dynamic vision sensor (DVS) detects temporal contrast of brightness and has the fastest response time compared to conventional frame-based sensors which detect static brightness per every frame. It can estimate the distance from DVS to an object by analyzing the spatial information of the reflection of additional light source. It also uses a pattern recognition based on time domain analysis of the reflection during turning on of the light source to avoid wrong proximity detection by noises such as other light sources and motions.

\section{2D HOLOGRAM PROJECTOR}

A 2D hologram projector is an optical device that projects an image on to a surface commonly a projection screen [5]. Most projectors create an image by making a light fall through a small transparent lens. The lens design and its attributes match the holographic area. The resulting effect is that of a free space display, because the image carrier appears very transparent.

\section{DYNAMIC VISION SENSOR}

The conventional sensors process series of frames continuously which is in efficient because it contains redundant information, wasting energy computational power and time. Use of DVS this drawback is eliminated [6] [3]. DVS starts to process the frames only when any change is sensed only at the change occurs. DVS in contrast works like human retina, causes only the local pixel level changes are transmitted [7] [8]. Power is drastically reduced whereas data storage and computational speed are drastically increased.

\section{PATTERN MATCHING SYSTEM}

The pattern matching is the act of checking a given sequence of tokens for the presence of the constituents of some pattern. In contrast to pattern recognition, the match usually has to be exact [4]. The patterns generally have the form of either sequences or tree structures. Uses of pattern matching include outputting the locations of a pattern within a token sequence, to output some component of the matched pattern, and to substitute the matching pattern with some other token sequence.

\section{PROPOSED METHOD}

The contributions of the proposed design are in three parts. First, it calculates accurate distance in real time only with spatial information of the reflection. Second, the proposed design can eliminate environmental noises by using pattern matching based on time domain analysis while conventional optical proximity sensors, which are mainly used in Smart phone, are very sensitive to environmental noises due to that they use the 


\section{Dynamic Vision Sensor based high efficiency Device Control}

total amount of brightness for certain period. Third, our design replaces conventional proximity sensors with holding additional benefits that it utilizes the advantages of DVS.

In this mechanism, we are going to use two dimensional hologram technologies to control specific devices. A design of proximity sensor utilizing DVS is proposed in this paper. The 2D hologram image is efficiently handled by the use of DVS, which is explained by its function as mentioned above. Light signal is focused by using the hologram projector, so that we can create the hologram image through 2D hologram projector. The projected hologram image will be monitored by the virtual sensing unit. Before making a serial communication via sensor medium access control unit, we need to process the image by using controlling unit. The micro control unit is used to process the image data and to control the devices through the switching unit. Hologram processing section is shown in Fig. 1.

The advantages of this proposed work is hardware usage can be reduced, power consumption will be low, easy to replace the controlling unit and it increases the processing efficiency and speed.

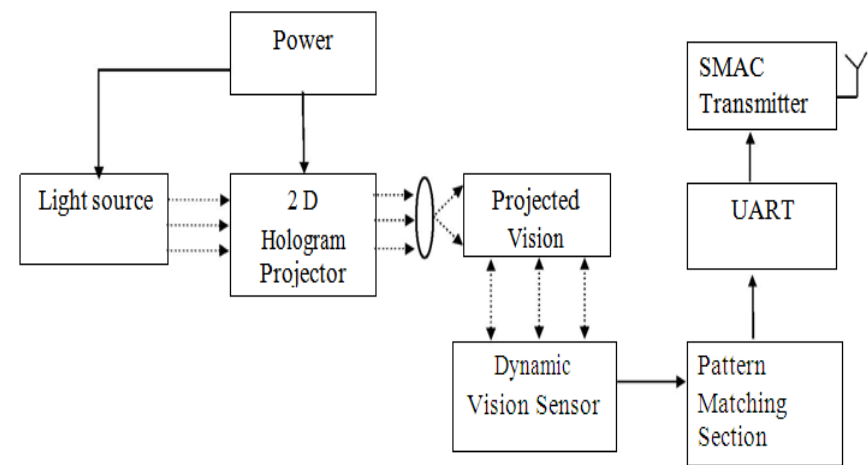

Fig. 1. Hologram Processing Section [Transmitter]

The microcontroller unit is used to process the image data and to control the devices through the switching unit and the following Fig. 2 shows device controlling unit.

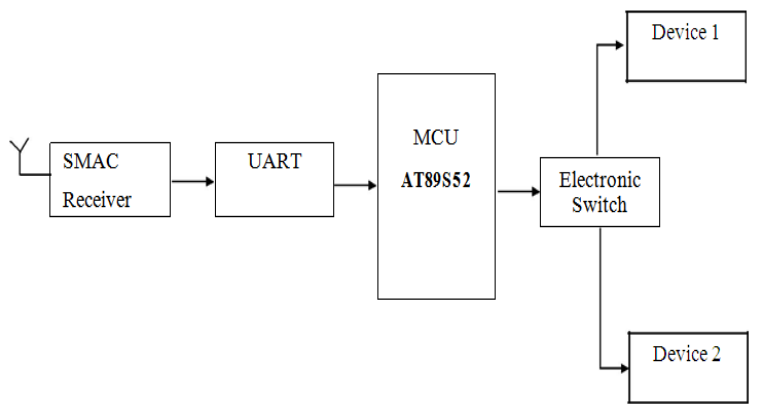

Fig. 2. Device Controlling Unit [Receiver]

\section{RESULT}

The mechanical set up of this project actually consists of the hologram projector and the project kit having transmitter and receiver section. The kit is connected to a port in the laptop or personal computer via USB cable from the UART port section of the kit and the port of the pc to which the USB cable is connected, is specified in the USB to serial application installed in the $\mathrm{PC}$. The hologram projector is connected to the port in the PC. The PC consists of the coding required to make the conditions needed for the device control via $2 \mathrm{D}$ hologram image. The transmitter section involves hologram processing and monitoring the processed image via dynamic vision sensor. This section consists of power supply required for the transmitter and receiver section and microcontroller unit. The communication between the transmitter and receiver section is of serial communication and is established by the use of MAX 232.

Once serial communication is established via sensor medium access control unit, the image needs to be processed by controlling unit. The microcontroller unit is used to process the image data and to control the devices through the switching unit. The receiver unit consists of ULN2003A IC, indicating LEDs and relays. There is also a section present that controls both the transmitter and receiver section.

The hologram is illuminated via LED and the image will be projected on the white screen. The illuminated image is projected over a screen and the DVS will be sensing the image. If the image that appeared is unaffected then the devices will also remain in the idle position i.e., device remains in off position. For no change in the input image projected, the device remains in off position. The image contrast remains the same and hence there will be no corresponding output.

Condition 1: The hologram is illuminated via LED and the image will be projected as shown in Fig. 3.

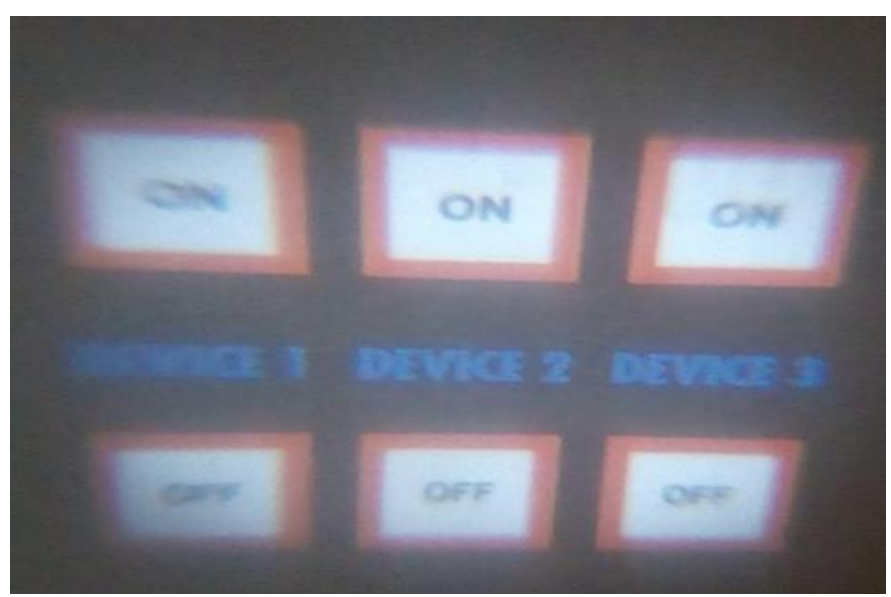

Fig. 3. Illuminated hologram image

The illuminated image is projected over a screen and the DVS will be sensing the image. If the image appeared unaffected then the devices will also remain in the idle position i.e., the led remains in off position. For no change in the input image projected, the device remains in off position as shown in Fig. 4. The image contrast remains the same and hence there will be no corresponding output. The following figure shows the resultant for condition 1 and also applicable to switch off condition for the device. The same is applied to off condition of the device. When the switch image OFF that lies below to the switch image $\mathrm{ON}$, is touched dynamic vision sensor detects the change in the contrast of the illuminated image via serial communication the change in image conveyed to microcontroller unit. 
Correspondingly the relay 1 is made open and hence the devise 1 is switched OFF. Any no. of relays can be connected and devise control can be achieved as per above mentioned procedure.

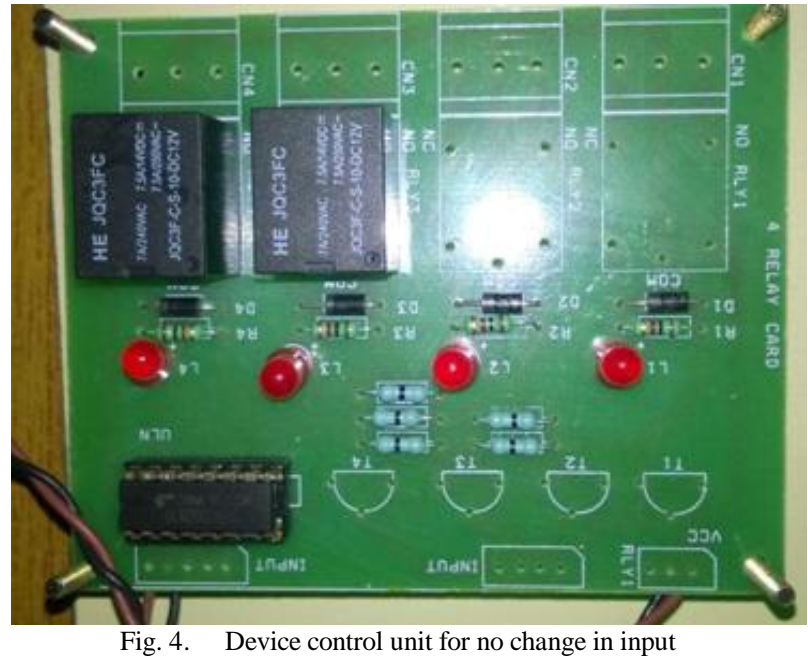

Condition 2: If the first led needs to be switched on, then the first row first switch image is touched, as shown in Fig. 5. The image contrast gets diminished when the shadow falls correspondingly the pixel value gets changed.

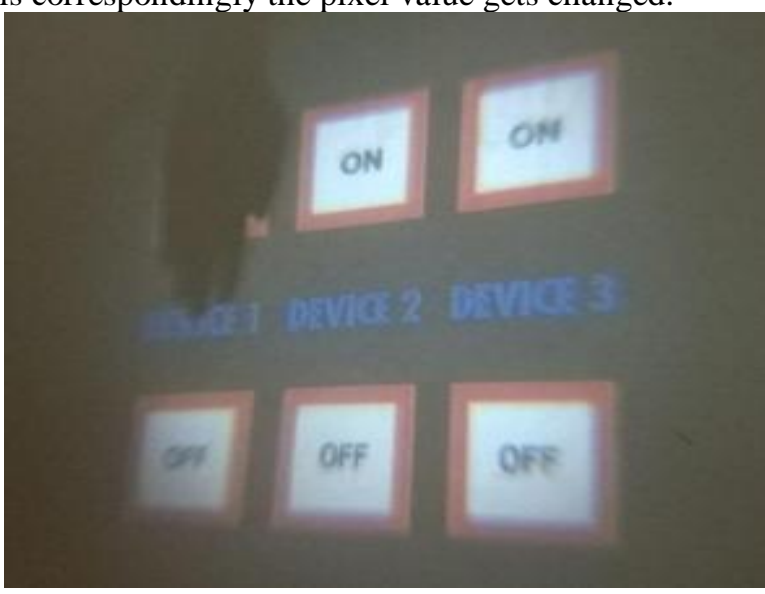

Fig. 5. Input control for first led

Dynamic vision sensor detects the change in the contrast of the illuminated image. And then serial communication is established via Universal asynchronous receiver transmitter. Then the microcontroller unit processes the image data and the switching unit controls the devices and the led is turned ON as shown in the Fig. 6.

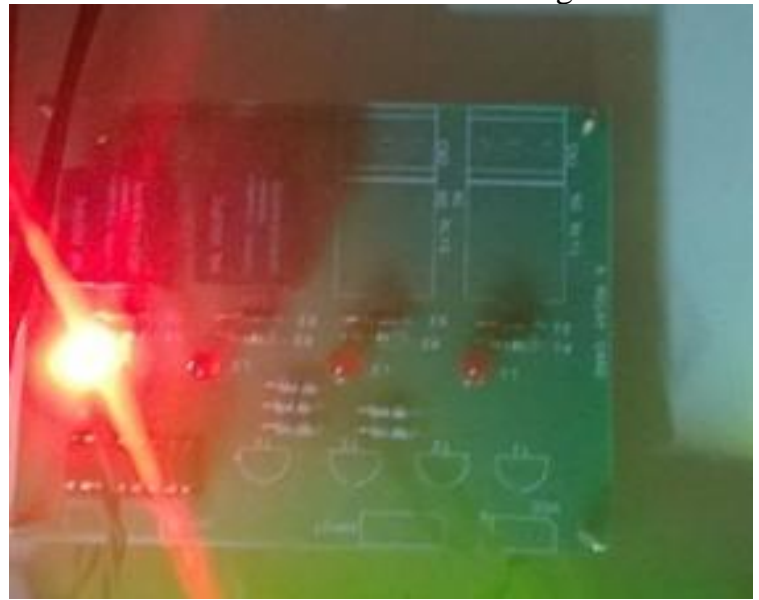

Fig. 6. LED turns on for the change in contrast over switch 1
The following is the Fig. 7 illustrates the graphical representation of signal to noise ratio vs. light density which is obtained from the data available from the Table I. One of the advantages of Dynamic Vision Sensor is high signal to noise ratio.

TABLE I. SNR VS. LIGHT DENSITY

\begin{tabular}{|c|c|c|}
\hline \multirow[b]{2}{*}{$\begin{array}{c}\text { LIGHT } \\
\text { DENSITY }\end{array}$} & \multicolumn{2}{|c|}{ SNR RATIO } \\
\hline & $\begin{array}{c}\text { PROXIMITY } \\
\text { SENSOR }\end{array}$ & $\begin{array}{c}\text { DVYNAMIC } \\
\text { VISION } \\
\text { SENSOR }\end{array}$ \\
\hline 0.2 & 0.4 & 0.7 \\
\hline 0.4 & 0.7 & 1.5 \\
\hline 0.6 & 1.2 & 2 \\
\hline 0.8 & 1.5 & 2.5 \\
\hline 1.0 & 1.8 & 3 \\
\hline 1.2 & 2.3 & 3.5 \\
\hline 1.4 & 2.5 & 4.1 \\
\hline
\end{tabular}

Absolute sensitivity threshold is the number of photons required to get a signal which is equivalent to the noise observed by the sensor. Absolute sensitivity threshold will be attained by IMX249 at a lower lighting level. IMX249 offers excellent image quality even in low light conditions and extraordinarily low noise performance.

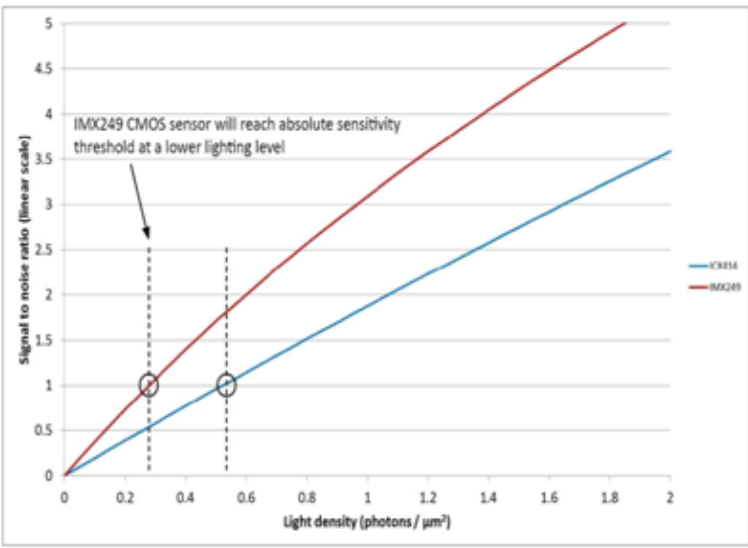

Fig. 7. Signal to noise ratio vs. light density

\section{CONCLUSION}

The results of this project provide an overview that this method has the following advantages: This method of control is wireless, Applicable to both domestic (home) and industrial related control of devices. In the current situation this current set-up only limited range of control radius is possible. Since it consumes less power and saves time by reducing the complexity of switch replacement, it may become reliable in the upcoming future. 
The microcontroller used in these setup costs less is an added advantage. Controlling is easily replaceable and hence usage becomes further convenient. The arrangement used for this method is wireless and this is an added advantage. The wireless receiver used in here is limited to small coverage area. But in feature it can be expanded in the radius level of the coverage if further advancements is proposed and put into practice after going through trial and error method.

In the upcoming future, enhanced range for the usage of this technology to achieve device control may become possible. Device control using 3D image can be done though it would be expensive. Multi screens of images can be made to appear by means of cascading of images enabling multi control of devices at a time. It is applicable to both domestic and industrial related control of devices. Since it consumes less power and saves time by reducing the complexity of switch replacement, it may become reliable in the upcoming future.

With just the controller language and program coding the functions can be implemented and if necessary, it can also be modified according to needs. In the coming future this technology might be useful in terms of reducing the need of hardware switches and enables the use of hologram projector in its place which would be further developed with virtual reality. A further enhancement could be made to the hologram technology associating it with the concept of virtual reality this would become the most efficient way of devise control in terms of commercial, domestic and industrial activities.

Any implementations for advancements is indeed expensive in the field of hologram, image processing based in virtual reality. If this technology gets stabilized, the cost might go down and it may become affordable in the future.

\section{REFERENCES}

[1] E. Buckley (2008) "Holographic laser projection technology (invited article)", Inf. Display, vol. 24, no. 12, pp. 22-25.

[2] R. Y. M. Chi. et al., (2008) "CMOS camera with in-pixel temporal change detection and ADC", IEEE J. of Solid-State Circ., vol. 43, no. 10, 2187- 2196.

[3] T. Delbrück. et al., (2010) "Activity-driven, event-based vision sensors," Proc. of the IEEE Int. Symp. on Circ. and Syst. (ISCAS), pp. 2426 - 2429.

[4] J. Salvi, J. Pags and J. Batlle (2004) "Pattern codification strategies in structured light systems", Pattern Recognit., vol. 37 , no. 4 , pp. $827-849$.

[5] T.-C. Poon. et al., (1993) "Real-time two-dimensional holographic imaging by using an electron-beam-addressed spatial light modulator", Opt. Lett., vol. 18, pp. 63.

[6] E. Y. Ahn. et al., (2011) "Dynamic vision sensor camera based bare hand gesture recognition", Proc. IEEE Symp. Comput. Intell. Multimedia, Signal Vis. Process., pp. 52-59.

[7] H.T. Cheng. et al., (2011) "Contactless gesture recognition system using proximity sensors", Proc. IEEE Int. Conf. Consum. Electron, pp. 149-150.

[8] W. Wei and A. Yunxiao (2009) "Vision-based human motion recognition: A survey", Proc. 2nd Int. Conf. Intell. Netw. Intell. Syst., pp. 386-389.

\section{AUTHORS PROFILE}

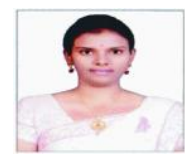

Ms. PRAVEENA. B completed B.E. Electronics and Instrumentation in R.M.D. Engineering College and M.E. Applied Electronics in R.M.K. Engineering College. She joined as an Assistant Professor in R.M.K. Engineering College in Electronics and Instrumentation Department. She has published papers in 3 international conference and 2 international journals in the technologies like Internet of Things and Thermo Electric Generator. She is a member of ISOI, ISTE. Currently, she is pursuing $\mathrm{PhD}$ with the area of interest as Internet of Things. She has attended and presented a paper in CVRDE Seminar with her students. She handled training session for students on Building Management System and Internet of Things.

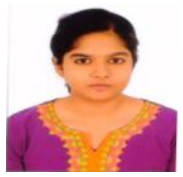

Ms. SHRAVANI $\mathbf{H}$ is pursuing B.E. Electronics and Instrumentation Engineering in R.M.K. Engineering (third year). She has secured 8.97 CGPA $^{*}$ as of $4^{\text {th }}$ semester. She has completed an electronics mini project and has also presented a paper on "Smart Irrigation System".

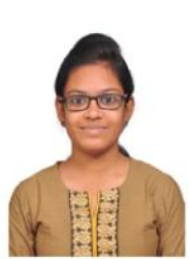

Ms SHIVANI A is pursuing $3^{\text {rd }}$ year B.E. Electronics and Instrumentation Engineering in R.M.K Engineering College. She has a Gpa of 8.32 (as of $4^{\text {th }}$ semester). She has a hands on experience in the project "Smart Irrigation System on IoT". She has also presented papers on the topic, "Content based Image Retrieval System over Hadoop using MapReduce and Smart Irrigation system using IoT”. 